\title{
Erratum zu: Zur Entwicklung domänenverbundener und domänenspezifischer Kompetenz im Ausbildungsverlauf
}

\author{
Eine Analyse für die kaufmännische Domäne
}

\section{Viola Katharina Klotz $\cdot$ Esther Winther}

Online publiziert: 2. Dezember 2016

(C) Springer Fachmedien Wiesbaden 2016

\section{Erratum zu:}

\section{Z Erziehungswiss (2016) 19:765-782}

DOI 10.1007/s11618-016-0687-1

Auf Grund eines technischen Fehlers wurden die Überschriften von Tab. 3 und 4 vertauscht. Für diesen Fehler bitten wir die Autorinnen sowie die Leser um Entschuldigung. Anbei die korrekten Tabellen.

Tab. 3 Domänenspezifische Kompetenz im Ausbildungsverlauf

\begin{tabular}{|c|c|c|c|c|c|}
\hline Subgruppe & $\mathrm{I}_{\mathrm{T}}$ & Fähigkeitsschätzer $(\theta)$ & Standardfehler & Chi-Square (df) & $p$-Wert \\
\hline \multicolumn{6}{|c|}{ Domänenspezifische Kompetenz } \\
\hline $\begin{array}{l}\text { Auszubildende zu Ausbil- } \\
\text { dungsbeginn }\end{array}$ & 1 & $-0,721$ & 0,016 & $3601,33(2)$ & 0,000 \\
\hline $\begin{array}{l}\text { Auszubildende nach } \\
0,5-1,5 \text { Jahren }\end{array}$ & 2 & $-0,325$ & 0,008 & - & - \\
\hline $\begin{array}{l}\text { Auszubildende nach } \\
1,7-2,8 \text { Jahren }\end{array}$ & 3 & 1,046 & 0,018 & - & - \\
\hline
\end{tabular}

Die Onlineversion des Originalartikels finden Sie unter DOI 10.1007/s11618-016-0687-1.

Prof. Dr. V. K. Klotz $(\bowtie)$

Fakultät für Betriebswirtschaftslehre, Universität Mannheim, L4, 1, 68161 Mannheim, Deutschland

E-Mail: viola.klotz@bwl.uni-mannheim.de

Prof. Dr. E. Winther

Leibniz-Zentrum für Lebenslanges Lernen e. V., Deutsches Institut für Erwachsenenbildung,

Heinemannstr. 12-14, 53175 Bonn, Deutschland

E-Mail: winther@die-bonn.de 
Tab. 4 Domänenverbundene Kompetenz im Ausbildungsverlauf

\begin{tabular}{|c|c|c|c|c|c|}
\hline Subgruppe & $\mathrm{I}_{\mathrm{T}}$ & Fähigkeitsschätzer $(\theta)$ & Standardfehler & Chi-Square (df) & $p$-Wert \\
\hline \multicolumn{6}{|c|}{ Domänenverbundene Kompetenz } \\
\hline $\begin{array}{l}\text { Auszubildende zu Ausbil- } \\
\text { dungsbeginn }\end{array}$ & 1 & $-0,506$ & 0,014 & $1575,35(2)$ & 0,000 \\
\hline $\begin{array}{l}\text { Auszubildende nach } \\
0,5-1,5 \text { Jahren }\end{array}$ & 2 & 0,104 & 0,007 & - & - \\
\hline $\begin{array}{l}\text { Auszubildende nach } \\
1,7-2,8 \text { Jahren }\end{array}$ & 3 & 0,402 & 0,015 & - & - \\
\hline
\end{tabular}

